\title{
Students' self-perceptions of mindfulness after learning mindfulness techniques in a professional skills course
}

\author{
Dara Dirhan, Alessandra Sarcona \\ Department of Nutrition, West Chester University of Pennsylvania, United States of \\ America.
}

\begin{abstract}
In this paper, we describe the effect of incorporating two mindfulness techniques (keeping a diary and motivational interviewing) in a professional skills course, and the impact these had on students' self-perceptions of mindfulness. Over a fourteen-week semester, students were asked to keep a diary at four different time points and were also taught motivational interviewing skills, which they had to apply to a four-part counseling project. Using a pre-post study design and the validated tool, the Mindful Attention Awareness Scale (MAAS), we measured students' perceptions of mindfulness pre-course and post-course. Results from the study indicated that students' perceptions of mindfulness significantly increased from pre-course to postcourse. Further, most students agreed that both the diary assignments and practicing motivational interviewing increased mindfulness from pre-course to post-course. Additionally, a majority of students reported that practicing motivational interviewing enhanced their counseling skills on the four-part counseling project and that the four diary assignments improved their writing skills. This study underscores the importance of incorporating mindfulness techniques into a professional skills course to increase students' mindfulness, which can further benefit the student by increasing students' professional skills to become more competent counselors and writers.
\end{abstract}

Keywords: Mindfulness; anxiety; diary; motivational interviewing; counseling; writing skills. 


\section{Introduction}

\subsection{Background on Mindfulness}

Over 40 million adults in the United States suffer from an anxiety disorder ("Understanding the facts," 2020). College students have substantially higher rates of anxiety and depression than the general population (Ibrahim et al., 2013). Stress and anxiety are within the top five health concerns for college students, which can lead to academic, mental, and emotional struggles (Griffin et al., 2020). College is a stressful time in the life of a young adult, yet there is evidence that practicing mindfulness can decrease symptoms of anxiety and depression (Martin, 2018). Kabat-Zinn (1994) defined mindfulness as, "paying attention in a particular way, on purpose, non-judgementally, to the present moment" (p. 4). Martin (2018) found that practicing mindfulness can increase well-being and decrease emotional reactivity among college students. Mindfulness is considered to be a disposition that can be enhanced by practicing mindful techniques (Sohl et al., 2016). Learning and practicing mindfulness techniques can further benefit college students because these practices can increase resilience to academic stressors and can enable students to more easily accept the ebb and flow of academic life (Ramasubramanian, 2017).

A meta-analytic evaluation of stress reduction interventions for university students indicate that cognitive-behavioral therapy, coping skills, and social support interventions were effective in reducing perceived stress, whereas relaxation training, mindfulness-based stress reduction, and psychoeducation were more effective in reducing anxiety (Yusufov, et al. (2019). Pogrebtsova et al. (2017) found that brief and cost-effective interventions focused on mindfulness techniques can quell stress and foster positive functioning. Creating brief, occasional diary entries is one such cost-effective mindfulness intervention that can be used among college students. This technique can incorporate a focus on social support from the reviewer. Interventions that employ social support provide environments where individuals are encouraged to communicate their experiences, thoughts, and feelings to one another (Kim et al., 2016) and this can be done through diary writing. Social support can buffer the impact of stress on numerous outcomes (Uchino \& Birmingham, 2011; Yusufov et al., 2019). Electronic diary entries can be especially beneficial to increase mindfulness in college students and to decrease anxiety, (Doorley et al., 2020). Expressive writing is a key component of journaling in a diary and is a commonly used tool for self-reflection (Frattaroli, 2006). This informal type of writing can give students a safe context to recognize their feelings on events in their lives so that they can gradually understand, validate, and accept them (Greenberg \& Lepore, 2004).

Another technique that can increase mindfulness is motivational interviewing (MI). MI is a counseling technique that embraces a spirit of supporting autonomy, eliciting one's reasons for change, and aligns with an assessment of readiness to change a behavior (Sohl et al., 
2016). MI leads to self-discovery, which is a component of mindfulness. Mindfulness influences client-centeredness, which is the main driver of MI (Sohl et al., 2016). Clinicians and counselors are also susceptible to job stress and burnout due to the high levels of empathetic demand that can create compassion fatigue or emotional exhaustion. Modified mindfulness training may help support clinician health, reduce burnout, improve job satisfaction and emotional well-being, which may have implications for patient care (Fortney et al., 2012). In addition, training clinicians to use MI has been shown to also reduce burnout scores (Pollak et al., 2016).

\subsection{Course assignments to increase mindfulness}

To assess students' perceptions of mindfulness from pre-course to post-course, two assignments focused on increasing mindfulness were created as required submissions in the professional skills course. A 4-part diary assignment was devised based on a twofold approach: first, as an emotional regulation technique to increase mindfulness, and second to improve students' writing skills. Bryant (1997) said that "you can't read and write well unless you can think straight, and you can't think straight unless you get in touch with your real thoughts" (p. 20). High levels of social anxiety correlate with increased difficulty in emotional regulation (Hayes-Skelton \& Graham, 2013). A study by Kivity and Huppert (2016) found that the practice of keeping a diary decreased social anxiety and increased emotional regulation. Based on this, the 4-part diary assignment was low stakes grading and provided points just for completion. Additionally, the style of writing was informal and encouraged free expression, so students would not feel judged but provided them with social support from the instructors. A 4-part nutrition counseling project was the second assignment with the twofold intent for students to practice MI skills, acting as the nutrition counselor to a client, and thereby increase their mindfulness. The counseling project required students to establish rapport with the client and to create goals and an interventional plan of action with the client in the spirit of MI. After three counseling sessions with their clients, students reflected on their MI skills, and changes in their self-efficacy, and mindfulness.

\subsection{Hypothesis and research questions}

We hypothesized that mindfulness would increase among the students from pre-course to post-course after students had learned about mindfulness techniques and given ample opportunity to practice some of the mindfulness techniques (i.e, diary entries and MI) throughout the semester. In addition, we expected that students' writing skills would improve as a result of practicing these mindfulness techniques in the professional skills course. Five research questions were formulated to guide our study; see Figure 1. 


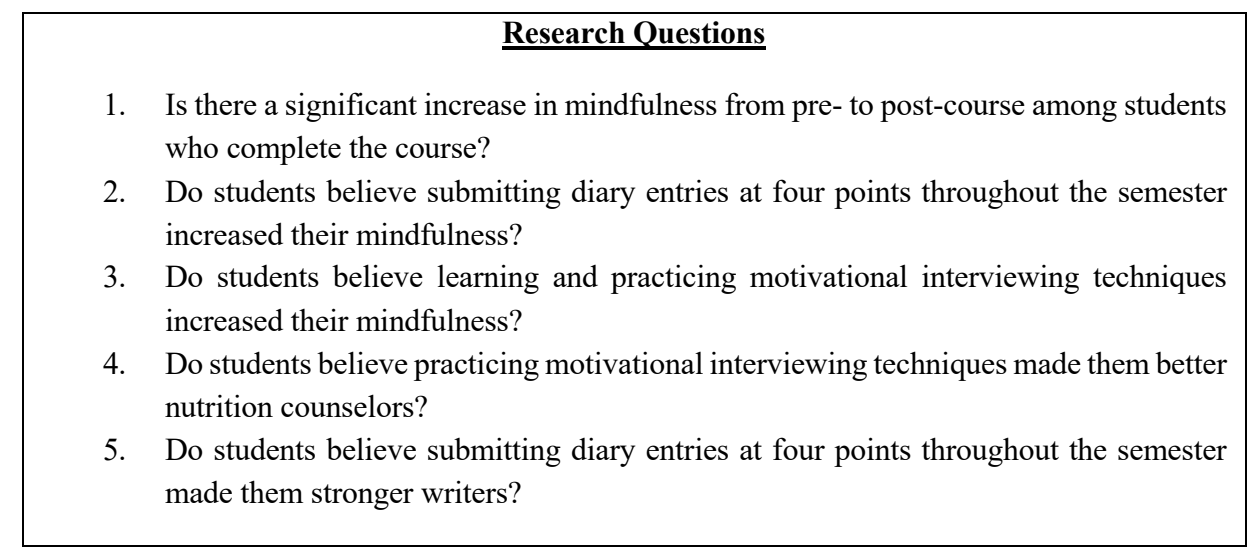

Figure 1. Research questions.

\section{Methods}

This pre-and post-test study was approved by the Institutional Review Board (IRB) of a public university located in the western suburbs of Philadephia and was introduced in a Professional Skills in Dietetics course. Throughout the semester, the instructors employed the use of a variety of mindfulness techniques (i.e diary entries and motivational interviewing skills) in the form of two, four-part assignments: a diary assignment, and a nutrition counseling project. The instructors provided lectures on the rationale behind diary entries making students stronger writers, and on MI skills to use in counseling clients. Instructor feedback was provided to the students on all assignment submissions.

\subsection{Participants}

A total of 102 third- and fourth-year students across three-course sections taught by two instructors for the course consented to participate in the study. All study participants were in the Nutrition and Dietetics major at the university and enrolled in the Professional Skills in Dietetics course. The study employed a convenience sample and participation in the study was voluntary. IRB approval was received before the start date of the study. There were no benefits to participating in the study, aside from furthering the existing body of literature on the topic of mindfulness.

\subsection{Data collection and analysis}

The students completed a 17-question survey as a pre-test on the first day of class, and they completed a 21-question post-test on the last day of class. Both the pre-test and the post-test employed the validated tool called the Mindful Attention Awareness Scale (MAAS). The MAAS is a 15-item scale used to study dispositional mindfulness. Items in the MAAS are consistent with the mindfulness theories on self-awareness (Bishop et al., 2004) and evaluate 
behaviors related to internal and external present-moment experiences. Additionally, questions on the post-test asked about students' perceptions of the correlations between the diary assignments and mindfulness, practicing motivational interviewing techniques and mindfulness, practicing MI techniques and being a better nutrition counselor to a client, and the diary entries effect on being a stronger writer.

Paired t-tests were used to compare students' perceptions from pre-test to post-test and frequencies were calculated using the Statistical Package for the Social Sciences (SPSS) version 22 to analyze students' perceptions of assignments throughout the course. The MAAS allows the researcher to calculate a dispositional mindfulness score based on how each participant assessed the fifteen survey items. A mean score can be calculated and higher scores reflect higher levels of dispositional mindfulness. As such, we calculated mean scores pre-course and post-course for the entire group of study participants.

\section{Results}

One hundred two students completed the pre-and post-tests and thereby completed the professional skills course that incorporated mindful activities. After computing mean frequencies on the MAAS for participants from pre-test to post-test, it was found that the mean mindful score significantly increased from pre-course to post-course. These results can be seen in Table 1.

Table 1. Mean Mindful Scores from Pre- to Post-Course.

\begin{tabular}{c|c|c}
\hline Mindful Score & Mean & Standard Deviation \\
\hline Pre-Course & 53.9 & 11.5 \\
Post-Course & 56.1 & 11.5 \\
\hline $\mathrm{N}=102, \mathrm{p}=.043$ & \multicolumn{2}{|l}{}
\end{tabular}

Frequencies were calculated to determine students' perceptions of mindfulness correlated to assignments throughout the course. When analyzing students' perceptions that the four-part diary assignments increased their mindfulness, it was found that the majority of students (91.2\%) slightly agreed to strongly agree that the diary assignments increased their mindfulness; see Table 2.

Table 2. Diary Assignment and Mindfulness Correlations.

\begin{tabular}{lcc}
\hline Response & Frequency & Percent \\
\hline 1. Strongly Disagree & 2 & 2.0 \\
2. Disagree & 3 & 2.9 \\
3. Slightly Disagree & 4 & 3.9 \\
4. Slightly Agree & 18 & 17.6 \\
5. Agree & 47 & 46.1 \\
6. Strongly Agree & 28 & 27.5 \\
\hline
\end{tabular}


Frequencies were calculated to determine students' perceptions of mindfulness, as correlated to learning about and having had the opportunity to practice motivational interviewing. Results indicated that almost all the students (99\%) slightly to strongly agreed that practicing motivational interviewing increased mindfulness; see Table 3.

Table 3. Practicing Motivational Interviewing and Mindfulness Correlations.

\begin{tabular}{lcc}
\hline Response & Frequency & Percent \\
\hline 1. Strongly Disagree & 0 & 0.0 \\
2. Disagree & 0 & 0.0 \\
3. Slightly Disagree & 1 & 1.0 \\
4. Slightly Agree & 25 & 24.5 \\
5. Agree & 39 & 38.2 \\
6. Strongly Agree & 37 & 36.3 \\
\hline
\end{tabular}

Frequencies were calculated to determine students' perceptions of the correlation between practicing motivational interviewing and their counseling skills. Results indicated that all the students slightly to strongly agreed that practicing motivational interviewing improved their counseling skills; see Table 4.

Table 4. Practicing Motivational Interviewing and Counseling Skills Correlations.

\begin{tabular}{lcc}
\hline Response & Frequency & Percent \\
\hline 1. Strongly Disagree & 0 & 0.0 \\
2. Disagree & 0 & 0.0 \\
3. Slightly Disagree & 0 & 0.0 \\
4. Slightly Agree & 3 & 2.9 \\
5. Agree & 32 & 31.4 \\
6. Strongly Agree & 65 & 65.7 \\
\hline
\end{tabular}

Frequencies were calculated to determine students' perceptions of the correlation between the completion of the diary assignment and their writing skills. The majority of students (89.2\%) slightly to strongly agreed that completing the diary assignments improved their writing skills; see Table 5.

Table 5. Diary Assignment and Writing Skills Correlations.

\begin{tabular}{lcc}
\hline Response & Frequency & Percent \\
\hline 1. Strongly Disagree & 1 & 1.0 \\
2. Disagree & 3 & 2.9 \\
3. Slightly Disagree & 7 & 6.9 \\
4. Slightly Agree & 15 & 14.7 \\
5. Agree & 37 & 36.3 \\
6. Strongly Agree & 39 & 38.2 \\
\hline
\end{tabular}

\section{Discussion}

Most of the research on mindfulness in college students has focused on meditation-based interventions. These studies have demonstrated an increase in mindfulness as measured by 
the MAAS when college students were taught mindful techniques such as meditation (Crowley et al., 2020; Shapiro et al., 2008). Our study was unique in adding mindful practices that were relative to writing and counseling skills along with increasing mindfulness. Students shared many stressful situations in their diary entries, and the instructors' nonjudgmental feedback on this low stakes grading assignment provided social support which has been shown to help reduce perceived stress (Yusufov et al., 2019; Kim et al., 2016; Uchino \& Birmingham, 2011) Overall, adding mindful techniques (i.e., diary assignments and MI) within the course semester increased students' overall mindful attention awareness.

Most of the students found that diary entry assignments and practicing MI increased their mindfulness. The MAAS is consistent with the mindfulness theories that focus on selfawareness behaviors in the present moment (Bishop et al., 2004). The diaries provided a form of expressive writing that aids in self-reflection and emotional regulation (Frattaroli, 2006; Kivity \& Huppert, 2016). Further, the majority of students found that being more mindful helped them to be better nutrition counselors and strengthened their writing skills. When training students to be future counselors it is imperative to provide strategies that will enable them to balance their empathy for their clients with their self-preservation. The study by Fortney (2013) used the Mindfulness-Based Stress Reduction (MBSR) program that includes discussions, yoga, stretching, along with other evidence-based strategies to help improve clinicians' well-being to reduce burnout. A study that involved a coaching intervention to teach MI to all clinic staff found that the clinicians in the intervention group reported improvements in burnout scores, self-rated MI skills, and perceived team cohesion (Pollak et $a l ., 2016$ ). Our students found that practicing MI increased mindfulness and improved their counseling skills. A strength of the study was using the validated MAAS that is commonly used in the literature to measure mindfulness. Limitations of the study include not having a control group and not using evidence-based strategies such as meditation to increase mindfulness.

\section{Conclusion}

This pre-and post-test study demonstrated that incorporating mindful activities and assignments into a professional skills course can increase students' perceptions of mindfulness, which can further improve students' counseling skills and writing skills. The mindful techniques used in this study related to skills necessary for students to practice in the nutrition and dietetics field. Using more evidence-based strategies can be considered for training students for their future careers. 


\section{References}

Anxiety and Depression Association of America. (2020, August 16). Understanding the facts of anxiety disorders and depression is the first step. Retrieved from https://adaa.org/about-adaa/press-room/facts-statistics

Bishop, S. R., Lau, M., Shapiro, S., Carlson, L., Anderson, N. D., Carmody, J., . . . Devins, G. (2004). Mindfulness: A proposed operational definition. Clinical Psychology: Science and Practice, 11, 230-241. http://dx.doi.org/10.1093/clipsy.bph077Bryant, D. (1997). Ella Price's Journal: A Novel. New York, NY: The Feminist Press at CUNY.

Crowley, C., Kapitula, L. R., \& Munk. (2020). Mindfulness, happiness and anxiety in a sample of college students before and after taking a meditation course. Journal of American College Health. doi:10.1080/07448481.2020.1754839.

Doorley, J.D., Volgenau, K.M., Kelso, K.C., Kashdan, T.B., \& Shackman, A.J. (2020). Do people with elevated social anxiety respond differently to digital and face-to-face communications? Two daily diary studies with null effects. Journal of Affective Disorders. 276, 859-865. doi:10.1016/j.jad.2020.07.069.

Fortney, L, Luchterland, C, Zakletskaia, L, Zgierska, A, \& Rakel, D. (2013). Abbreviated mindfulness intervention for job satisfaction, quality of life, and compassion in primary care clinicians: A pilot study. Annals of Family Medicine, 11, 412-420. doi:10.1370/afm.1511.

Frattaroli, J. (2006). Experimental disclosure and its moderators: A metaanalysis. Psychological Bulletin, 132, 823-865. http://dx.doi.org/10.1037/0033-2909.132.6.823

Goodman, M.J., \& Schorling, J.B. (2012). A mindfulness course decreases burnout and improves well-being among healthcare providers. The International Journal of Psychiatry in Medicine. 43, 119-128. doi:10.2190/PM.43.2.b.

Greenberg, M. A., \& Lepore, S. J. (2004). Theoretical mechanisms involved in disclosure: From inhibition to self regulation. In I. Nyklicek, L. Temoshok, \& A. J. J. M. Vingerhoets (Eds.), Emotional expresión and health advances in theory, assessment and clinical applications (pp. 42-59). New York, NY: Brunner-Routledge.

Griffin, M., Campos, H.C., Khramtsova, I., \& Pearce, A.R. (2020). Stress and anxiety reduction in college students through biofeedback. College Student Journal. 54(2), 258268. Retrieved from https://projectinnovation.com/

Hayes-Skelton, S., \& Graham, J. (2013). Decentering as a common link among mindfulness, cognitive reappraisal, and social anxiety. Behavioural and Cognitive Psychotherapy, 41, 317-328.

Ibrahim, A.K., Kelly, S.J., Adams, C.E., \& Glazebrook, C. (2013). A systematic review of studies of depression prevalence in university students. Journal of Psychiatric Research. 47(3), 391-400. doi: 10.1016/j.jpsychires.2012.11.015.

Kabat-Zinn, J. (1994). Wherever you go, there you are: Mindfulness meditation in everyday life. New York, NY: Hyperion Books.

Kim, S., Lee, H., Kim, H., Noh, D., \& Lee, H. (2016). Effects of an integrated stress management program (ISMP) for psychologically distressed students: A randomized controlled trial. Perspectives in Psychiatric Care, 52, 178-185. http://dx.doi.org/10.1111/ppc.12114 
Kivity, Y., \& Huppert, J. D. (2016). Does cognitive reappraisal reduce anxiety? A daily diary study of a micro-intervention with individuals with high social anxiety. Journal of Consulting and Clinical Psychology, 84(3), 269-283. https://doi.org/10.1037/ccp0000075

Martin, M. (2018). Mindfulness and transformation in a college classroom. Adult Learning. 29(1), 5-10. doi: 10.1177/1045159517744752.

Pollak, K. I., Nagy, P., Bigger, J., Bilheimer, A., Lyna, P., Gao, X...Armstrong, S. (2016). Effect of teaching motivational interviewing via communication coaching on clinician and patient satisfaction in primary care and pediatric obesity-focused offices. Patient Education Counseling. 99(2), 300-303. doi:10.1016/j.pec.2015.08.013.

Pogrebtsova, E., Craig, J., Chris, A., O’Shea, D., \& Gonzalez, M.M.G. (2018). Exploring daily affective changes in university students with a mindful positive reappraisal intervention: A daily diary randomized controlled trial. Stress and Health: Journal of the International Society for the Investigation on Stress. 34(1), 46-58. doi: 10.1002/smi.2759.

Ramasubramanian, S. (2017). Mindfulness, stress coping and everyday resilience among emerging youth in a university setting: a mixed methods approach. International Journal of Adolescence and Youth. 22(3), 308-321. doi:10.1080/02673843.2016.1175361.

Shapiro, S., Oman, D., Thoresen, C. C., Plante, T. G., \& Flanders, T. (2008). Cultivating mindfulness: Effects on well-being. Journal of Clinical Psychology. 64(7), 840-862. doi:10.1002/jclp.20491

Sohl, S.J., Birdee, G., \& Elam, R. (2016). Complementary tools to empower and sustain behavior change: motivational interviewing and mindfulness. American Journal of Lifestyle Medicine. 10(6), 429-436. doi:10.1177/1559827615571524.

Yusufov, M., Nicoloro-SantaBarbara, J., Grey, N. E., Moyer, A., \& Lobel, M. (2019). Metaanalytic evaluation of stress reduction interventions for undergraduate and graduate students. International Journal of Stress Management, 26(2), 132-145. https://doi.org/10.1037/str0000099 